EXTENDED REPORT

\title{
Scoring of radiographic progression in randomised clinical trials in ankylosing spondylitis: a preference for paired reading order
}

\author{
A Wanders, R Landewé, A Spoorenberg, K de Vlam, H Mielants, M Dougados, \\ $S$ van der Linden, $D$ van der Heijde
}

See end of article for authors' affiliations

Correspondence to:

Dr R Landewé, Department of Internal Medicine,

Division of Rheumatology, University Hospital

Maastricht, PO Box 5800

6202 AZ Maastricht, The

Netherlands;

rlan@sint.azm.nl

Accepted 20 June 2004

Published Online First

5 August 2004

Objectives: To describe the influence of the reading order (chronological $v$ paired) on radiographic scoring results in ankylosing spondylitis. To investigate whether this method is sufficiently sensitive to change because paired reading is requested for establishing drug efficacy in clinical trials.

Methods: Films obtained from 166 patients (at baseline, 1 year, and 2 years) were scored by one observer, using the modified Stoke Ankylosing Spondylitis Spinal Score. Films were first scored chronologically, and were scored paired 6 months later.

Results: Chronological reading showed significantly more progression than paired reading both at 1 year (mean (SD) progression $1.3(2.6) \vee 0.5(2.4)$ units) and at 2 years (2.1 (3.9) $\vee 1.0$ (2.9) units); betweenmethod difference: $p<0.001$ at 1 year, and $p<0.001$ at 2 years. After 1 year, progression ( $>0$ units) was found in $35 / 166(21 \%)$ patients after paired reading and in 55/166 (33\%) after chronological reading. After 2 years, these figures were 50/166 (30\%) and 68/166 (41\%), respectively. Sample size calculations showed that 94 patients in each treatment arm are required in a randomised clinical trial (RCT) to provide sufficient statistical power to detect a difference in 2 year progression if films are scored paired.

Conclusion: Reading with chronological time order is more sensitive to change than reading with paired time order, but paired reading is sufficiently sensitive to pick up change with a follow up of 2 years, resulting in an acceptable sample size for RCTs.

$\mathrm{F}$ or evaluation of treatment in ankylosing spondylitis(AS), the ASessment in Ankylosing Spondylitis (ASAS) working group has developed core sets to be used in various settings, ${ }^{1}$ including the setting for disease controlling antirheumatic treatment (D-CART). One segment of the definition of D-CART reads: "prevent or significantly decrease the rate of progression of structural damage". ${ }^{1}$ To assess progression of structural damage, radiographic outcome assessment is included in the D-CART core set. Radiology as outcome measure in AS clinical trials is new, in contrast with clinical trials in rheumatoid arthritis (RA), in which radiographic outcome already has a prominent place. The methodology of radiographic scoring in AS is still developing. Recently, we performed a study comparing the existing radiographic scoring methods for various aspects of validity. It was concluded that the modified Stoke Ankylosing Spondylitis Spinal Score (modified SASSS) is the most appropriate method for use in clinical trials.

It is known from studies evaluating radiographic damage in RA that the order in which films are presented to the observer influences results. ${ }^{3-6}$ Films can be grouped for each patient and presented without the reader knowing the chronological order of the films: paired scoring. Films can also be grouped for each patient and presented in chronological order. The advantage of chronological reading is that it provides the reader with maximum information, thereby reducing "true" measurement error. Reading films chronologically increases the ability to detect changes in comparison with paired reading. In 1999 van der Heijde et al showed that reading with chronological order was more sensitive to change than paired reading in RA. ${ }^{5}$ However, the possibility that chronological reading overestimates progression of joint damage because readers expect to see progression (expectation bias) could not be excluded.
In a follow up study Bruynesteyn et al used progression considered as clinically relevant by rheumatologists as a proxy for true progression, and concluded that paired reading underestimates the true progression. ${ }^{6}$ The advantage of paired reading, however, is that expectation bias is almost ruled out: readers are not aware of the sequence of the films and therefore do not tend to score more progression in the follow up film. The question as to which of the two reading orders should be used is therefore not unanimously answered by the above-mentioned studies.

Despite this controversy, the reading of structural damage in RA clinical trials is predominantly performed by readers who are unaware of the sequence. This stems from the general epidemiological consensus that to prevent bias observers must be "blinded" as far as possible, and from the practical aspect that for registration purposes reading with "blinded" sequence is requested by the drug regulatory agencies. Therefore it seems obvious that radiographic progression in AS clinical trials should also be assessed by paired reading. However, there is some concern that paired scoring in AS is not sufficiently sensitive, because progression occurs slowly, and only in a minority of patients. ${ }^{7}$

This study, therefore, aimed at (a) exploring the differences in sensitivity to change between paired and chronological scoring in AS, and $(b)$ investigating whether trials with radiographic progression as a primary end point can be designed that have sufficient statistical power with feasible patient numbers if films are read with paired order.

Abbreviations: AS, ankylosing spondylitis; D-CART, disease controlling antirheumatic treatment; RA, rheumatoid arthritis; RCT, randomised controlled trial; SASSS, Stoke Ankylosing Spondylitis Spinal Score 
Table 1 Patient characteristics at baseline

\begin{tabular}{|c|c|c|c|c|c|}
\hline & Mean & SD & Median & $\begin{array}{l}\text { 25th } \\
\text { Centile }\end{array}$ & $\begin{array}{l}\text { 75th } \\
\text { Centile }\end{array}$ \\
\hline Age (years) & 43.9 & 12.5 & 43.1 & 33.6 & 52.9 \\
\hline Mean duration of complaints (years) & 20.4 & 11.6 & 17.1 & 12.0 & 27.5 \\
\hline Mean duration of disease after diagnosis (years) & 11.7 & 9.0 & 10.0 & 4.8 & 15.4 \\
\hline
\end{tabular}

\section{PATIENTS AND METHODS Patients and films}

Radiographs from an international longitudinal, observational study on outcome in AS, the OASIS cohort, were used. ${ }^{8}$ Originally, 217 patients from four centres in the Netherlands, Belgium, and France were included in this cohort. Radiographs were obtained at baseline, and after 1 and 2 years of follow up. After 2 years of follow up, complete sets of radiographs of baseline, 1 year, and 2 years of 166 patients were available; only these patients were included in this study. The modified SASSS was assessed on lateral views of the lumbar and cervical spine.

\section{Scoring of films}

The modified SASSS method scores every corner of the anterior site of the lumbar and cervical vertebrae on a scale from 0 to 3, in which 0 indicates no abnormalities; 1 indicates erosion, sclerosis, or squaring; 2 indicates a syndesmophyte; and 3 a bridging syndesmophyte. This yields a possible total score of 72 units. The lumbar spine is scored from the lower border of the 12th thoracic vertebra to the upper border of the first sacral vertebra. The cervical spine is scored from the lower border of the second cervical vertebra to the upper border of the first thoracic vertebra. In a previous study it was shown that this method had good inter- and intraobserver reliability. ${ }^{2}$ Intraclass correlation coefficients for inter- and intraobserver reliability for progression scores with a 2 year interval were 0.82 and 0.95 , respectively. Films were available for three times: baseline, 1 year, and 2 years. Firstly, the films were scored in chronological order, and after 6 months the films were scored again by the same reader (AW), but now in a random time order (paired films for each patient). The chronological scoring method allows negative progression scores.

\section{Analysis and statistics}

Descriptive statistics (mean, standard deviation, median, 25th and 75th centile) are given for the modified SASSS scores for both reading orders at the three times, as well as for the progression scores. Also, descriptive statistics are provided for those patients who had a radiographic progression greater than zero. To visualise the effects of scoring by the two reading orders, progression scores obtained by both methods were plotted by their cumulative frequency (expressed as percentage; cumulative probability) in probability plots. ${ }^{9}$ Wilcoxon's signed ranks test was used to test the null hypothesis that 1 or 2 year progression is zero. A Mann-Whitney test was used to investigate the null hypothesis that radiographic progression obtained by both reading orders was similar. Proportions of patients with progression ( $>0$ units) by reading order at 1 or 2 years were compared by $\chi^{2}$ test.

Sample sizes for a putative randomised controlled trial (RCT) with one untreated control group and one active treatment group, and radiographic progression as primary end point, were calculated using the power calculator of the University of California, Los Angeles (http://calculators.stat.ucla.edu/powercalc/ (accessed 12 September 2004), significance level 0.05, two sided, power 0.80). This was done under the assumption that an untreated control group will show progression as in the OASIS cohort, and that progression in the active treatment group is zero, with a standard deviation equal to the standard deviation in the untreated control group. Van der Waerden normalised progression scores were used to perform the sample size calculations.

\section{RESULTS}

\section{Sensitivity to change}

Table 1 shows the patient characteristics at baseline. In table 2 the descriptive statistics of the modified SASSS scores according to chronological and paired reading are given. Baseline scores are almost similar. Reading with chronological order yields more progression than paired reading, both at 1 year $(1.3$ (2.6) (mean (standard deviation)) $v 0.5(2.4)$ units) and at 2 years (2.1 (3.9) $v 1.0$ (2.9) units). Table 3 provides the descriptive statistics of the modified SASSS

Table 2 Descriptive statistics of 1 and 2 year follow up of radiological damage scored according to the modified SASSS with paired and chronological reading order $(n=166$ patients)

\begin{tabular}{lrrllr}
\hline & Mean & SD & Median & 25th Centile & 75th Centile \\
\hline Paired reading order & & & & & \\
Baseline & 13.1 & 18.0 & 4.9 & 0.0 & 18.1 \\
1 Year & 13.6 & 18.4 & 4.9 & 0.0 & 21.2 \\
2 Years & 14.1 & 18.6 & 6.0 & 0.0 & 23.3 \\
Progression after 1 year & 0.5 & 2.4 & 0.0 & 0.0 & 0.0 \\
Progression after 2 years & 1.0 & 2.9 & 0.0 & 0.0 & 1.6 \\
& & & & & \\
Chronological reading order & 13.6 & 19.2 & 5.0 & 0.0 & 17.0 \\
Baseline & 14.9 & 19.7 & 6.0 & 0.0 & 20.1 \\
1 Year & 15.8 & 20.1 & 6.0 & 0.0 & 22.7 \\
2 Years & 1.3 & 2.6 & 0.0 & 0.0 & 1.4 \\
Progression after 1 year & 2.1 & 3.9 & 0.0 & 0.0 & 3.0 \\
Progression after 2 years & & &
\end{tabular}

Modified SASSS, modified Stoke Ankylosing Spondylitis Spinal Score; SD, standard deviation. 
Table 3 Descriptive statistics of 1 and 2 year follow up of radiological damage scored according to the modified SASSS of the patients who showed progression greater than zero according to the paired and chronological reading order

\begin{tabular}{lllllll}
\hline & No & Mean & SD & Median & $\begin{array}{l}\text { 25th } \\
\text { Centile }\end{array}$ & $\begin{array}{l}\text { 75th } \\
\text { Centile }\end{array}$ \\
\hline $\begin{array}{l}\text { Paired reading order } \\
\text { Progression after 1 year }\end{array}$ & 35 & 4.0 & 2.9 & 4.0 & 1.6 & 5.0 \\
Progression after 2 years & 50 & 3.9 & 3.8 & 2.9 & 2.0 & 5.0 \\
& & & & & & \\
$\begin{array}{l}\text { Chronological reading order } \\
\text { Progression after 1 year }\end{array}$ & 55 & 3.9 & 3.1 & 3.0 & 1.2 & 6.0 \\
Progression after 2 years & 68 & 5.2 & 4.6 & 4.0 & 2.0 & 7.0 \\
\hline
\end{tabular}

scores of those patients who showed a progression greater than zero.

In the entire cohort of this study, both methods detected progression from baseline significantly (chronological order: $\mathrm{p}<0.001$ for 1 year, and $\mathrm{p}<0.001$ for 2 years; paired order: $\mathrm{p}=0.021$ for 1 year, and $\mathrm{p}<0.001$ for 2 years). Reading with chronological order was significantly more sensitive than paired reading (between-method difference: $p<0.001$ at 1 year, and $p<0.001$ at 2 years). After 1 year of follow up $35 / 166(21 \%)$ of patients showed progression $>0$ units according to the paired reading results and 55/166 (33\%) of patients according to the chronological reading results. At the 2 year follow up these figures were 50/166 (30\%) and 68/166 (41\%), respectively.

This progression pattern is further illustrated by probability plots for the 1 year (fig 1 ) and 2 year interval (fig 2). Figure 1 shows that for both scoring methods most patients do not show progression. This was already represented by the median, which was zero for both methods (this median value can be found on the $\mathrm{x}$ axis at a cumulative probability of 0.50 ) The advantage of the probability plot is that it also easily represents the percentage of patients with progression: for instance, fig 1 for the chronological reading order shows that the curve deviates from zero at a value of $67 \%$, indicating that $33 \%$ of patients show progression. Although negative progression scores were allowed in the chronological scoring method, these are not seen in the two plots. For the paired scoring method negative scores are visible in both figures ( $15 \%$ at 1 year and $11 \%$ at 2 years).

A comparison of both plots shows that the curve for chronological reading lies further to the left, which indicates that with the chronological reading more patients are classed

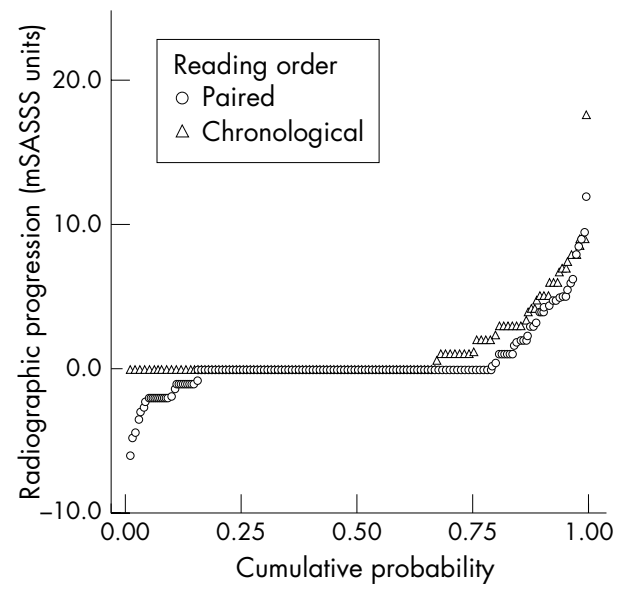

Figure 1 Probability plot of 1 year progression in modified SASSS scores for paired and chronological reading order. as progressive. The difference between these two curves was statistically tested; for both the 1 year interval and the 2 year interval the difference between both methods was significant $(\mathrm{p}=0.019$ and $\mathrm{p}=0.051$, respectively $)$.

Sample size calculations for the paired reading order Table 1 and the probability plots show that the data of the paired scoring order have a skewed distribution. So before entering the data in sample size calculations a van der Waerden normalisation procedure was performed. The following assumptions were made in the sample size calculations: the mean progression in the intervention group is zero and the standard deviation is the same as in the control group (the OASIS cohort). With these assumptions the following sample sizes were obtained for an RCT in which radiographic progression is scored according to the modified SASSS by paired reading order: an RCT with a duration of 1 year requires 922 patients in each arm, and an RCT with a duration of 2 years requires 94 patients in each arm, in order to statistically underline a true between-group difference of 0.5 units ( 1 year) or 1.0 units ( 2 years).

\section{DISCUSSION}

The conclusion of this study is that the order in which films of patients with AS are presented to the observer influences the reading results, which is in accordance with the findings in RA. Reading films in a chronological order shows a higher mean progression and a greater proportion of patients with progression, in comparison with paired reading.

However, we also showed that scoring with a paired reading order is sufficiently sensitive to detect radiographic progression after 2 years of follow up, under the specific

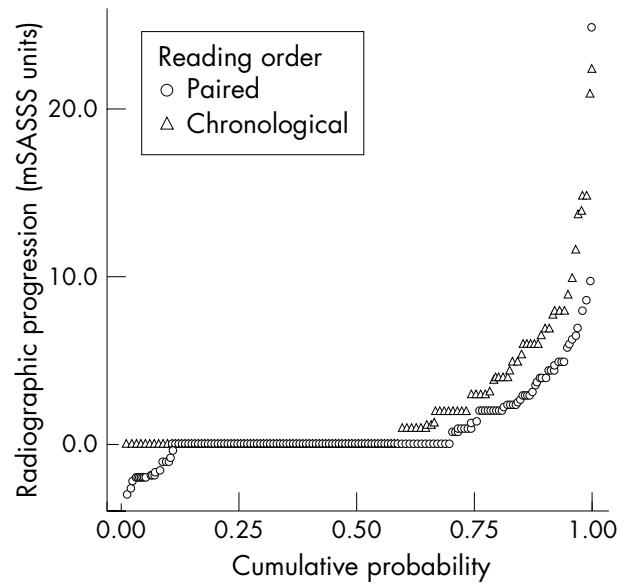

Figure 2 Probability plot of 2 year progression in modified SASSS scores for paired and chronological reading order. 
conditions set in this study. To illustrate the feasibility of paired scoring in trials with a radiographic end point, we demonstrated an acceptable sample size for a putative RCT, using real progression data from the OASIS cohort, provided that the duration of the trial is at least 2 years.

The theoretical assumption that chronological scoring in comparison with paired scoring would have a higher sensitivity to change, which is supported by data from research in RA, was confirmed in this study. It was also seen that the magnitude of the signal detected by the chronological reading order is greater than by the paired reading order. However, which part of this signal is a "true" effect and which part can be attributed to "noise" is difficult to establish, especially for the chronological reading order. First of all, in the chronological reading order expectation bias contributes to "noise", whereas this bias is almost ruled out in paired scoring. It is impossible to determine in chronological reading which part of "noise" is caused by expectation bias and which part by the remaining measurement error. The measurement error in paired reading can be visualised by means of probability plots. Because it is thought that the phenomenon of healing ("true negative scores") does not occur in AS (which is supported by the results of chronological reading data, in which no negative scores were found), the negative scores obtained by paired scoring can be considered as measurement error. When this is applied to fig 1 with a 1 year time interval, then it is seen that $15 \%$ of the patients have a negative score. The percentage of patients that have a positive score is $21 \%$. Assuming that measurement error works equally in both directions, this would mean that only $6 \%$ of patients show "real" progression. In fig 2, with a 2 year interval, it is seen that $11 \%$ of patients have a negative score and $30 \%$ of patients have a positive score, which means that $19 \%$ of patients show "real" progression. This difference in signal-noise ratio is also reflected by the sample size calculations, after 1 year of follow up a huge sample size is needed-922 patients, versus 94 patients for a follow up of 2 years.

The lack of expectation bias and the possibility of assessing measurement error are advantages of paired reading. Apart from these advantages, it is also a fact that this scoring method is requested by the agencies for registration purposes. Therefore the feasibility of this method is relevant with respect to the number of patients needed to demonstrate a significant difference in radiographic progression. The problem with sample size calculations is that they are dependent on the assumptions, which are arbitrary. Determining the assumptions underlying an RCT with radiographic progression in AS as an outcome measure is particularly difficult, because not much is known about the effect of interventions on radiographic progression. Data from a study in RA showed that anti-tumour necrosis factor treatment inhibited radiographic progression, ${ }^{10}$ which might support our assumption of zero progression. However, despite all the assumptions and uncertainties associated with sample size calculations, there is a precedent that shows that a sample size of 94 patients may provide sufficient statistical power. Recently an RCT in AS was performed in which radiographic progression of
2 years was used as the primary outcome measure. ${ }^{11}$ In this RCT radiographic progression for continuous versus on demand intake of non-steroidal anti-inflammatory drugs was compared. Radiographic progression was assessed by the modified SASSS with a paired scoring order. The two treatment groups comprised 74 and 76 patients, and a between-group difference of 1.1 was found to be statistically significant in this study.

Therefore, based on theoretical arguments and on the results of this study we recommend that RCTs in AS, with radiographic progression as an end point, should be of 2 years' duration, and should be scored by a paired reading order.

\section{Authors' affiliations}

A Wanders, $\mathbf{R}$ Landewé, A Spoorenberg, $\mathbf{S}$ van der Linden

D van der Heijde, Department of Internal Medicine, Division of Rheumatology, University Hospital Maastricht, The Netherlands $\mathrm{K}$ de Vlam, H Mielants, Department of Rheumatology, University Hospital Gent, Belgium

M Dougados, Department of Rheumatology, Hôpital Cochin Paris, France

\section{REFERENCES}

1 van der Heijde D, van der Linden S, Bellamy N, Calin A, Dougados M, Khan MA. Which domains should be included in a core set for endpoints in ankylosing spondylitis? Introduction to the ankylosing spondylitis module of OMERACT IV. J Rheumatol 1999;26:945-7.

2 Wanders A, Landewé R, Spoorenberg A, Dougados M, van der Linden S, Mielants $\mathrm{H}$, et al. What is the most appropriate radiologic scoring method for ankylosing spondylitis? A comparison of the available methods based on the outcome measures in rheumatology clinical trials filter. Arthritis Rheum 2004;50:2622-32.

3 Ferrara R, Priolo F, Cammisa M, Bacarini L, Cerase A, Pasero G, et al. Clinical trials in rheumatoid arthritis: methodological suggestions for assessing radiographs arising from the GRISAR Study. Gruppo Reumatologi Italiani Studio Artrite Reumatoide. Ann Rheum Dis 1997;56:608-12.

4 Salaffi F, Carotti M. Interobserver variation in quantitative analysis of hand radiographs in rheumatoid arthritis: comparison of 3 different reading procedures. J Rheumatol 1997;24:2055-6.

5 van der Heijde D, Boonen A, Boers M, Kostense P, van der Linden S. Reading radiographs in chronological order, in pairs or as single films has important implications for the discriminative power of rheumatoid arthritis clinical trials. Rheumatology (Oxford) 1999;38:1213-20.

6 Bruynesteyn K, van der Heijde D, Boers M, Saudan A, Peloso P, Paulus H, et al. Detecting radiological changes in rheumatoid arthritis that are considered important by clinical experts: influence of reading with or without known sequence. J Rheumatol 2002;29:2306-12.

7 Spoorenberg A, de Vlam K, van der Linden S, Dougados M, Mielants H, van de Tempel $\mathrm{H}$, et al. Radiological scoring methods in ankylosing spondylitis. Reliability and sensitivity to change over one and two years. J Rheumatol 2004;31:125-32.

8 Spoorenberg A, van der Heijde D, de Klerk E, Dougados M, de Vlam K, Mielants $\mathrm{H}$, et al. Relative value of erythrocyte sedimentation rate and $\mathrm{C}$ reactive protein in assessment of disease activity in ankylosing spondylitis. $J$ Rheumatol 1999;26:980-4.

9 Landewé $\mathbf{R}$, van der Heijde DFMF. Radiographic progression visualised by probability plots: presenting data with optimal use of individual values. Arthritis Rheum 2004;50:699-706.

10 Lipsky PE, van der Heijde DM, St Clair EW, Furst DE, Breedveld FC, Kalden JR, et al. Infliximab and methotrexate in the treatment of rheumatoid arthritis. Anti-Tumor Necrosis Factor Trial in Rheumatoid Arthritis with Concomitant Therapy Study Group. N Engl J Med 2000;343:1594-602.

11 Wanders A, van der Heijde D, Landewé R, Béhier J-M, Calin A, Olivieri I, et al. Inhibition of radiographic progression in ankylosing spondylitis by continuous use of NSAIDs [abstract]. Arthritis Rheum 2003;(suppl): abstr 518 . 